\title{
Experiencia de Innovación Docente: Turistización y Paisaje Histórico de Sevilla
}

\author{
DAVId SÁNCHEZ BEnÍTEZ \\ Departamento de Geografía Humana \\ Universidad de Sevilla \\ dsanchez5@us.es \\ ORCID: https://orcid.org/0000-0001-9581-2981 \\ D.O.I.: http://dx.doi.org/10.12795/JDU.2018.i01.41 \\ Pp.: $735-750$
}

\section{Resumen}

Se presentan en esta comunicación los resultados de la experiencia de innovación docente dentro de los Ciclos de Mejora Docente (CMD) desarrollada en la Red para la Formación y la Innovación Docente (REFID) para abordar la totalidad de los contenidos pertenecientes al tema 4 "El análisis de los paisajes y escenarios. Aproximaciones al análisis del paisaje desde distintas escalas" de la asignatura de primero de Grado de Geografía y Gestión del Territorio llamada Taller Práctico de Geografía Humana. Se plantean innovaciones en el modelo metodológico mediante el uso del taller conceptual intentando centrar la experiencia en quién aprende, el trabajo cooperativo y en escenarios lo más cercanos posible a la realidad del alumnado.

Palabras clave: Taller Práctico de Geografía Humana, Grado de Geografía y Gestión del Territorio, docencia universitaria, experimentación docente universitaria, paisaje y turistización. 


\section{Contexto de la intervención}

La actividad se diseñó para la asignatura cuatrimestral "Taller Práctico de Geografía Humana" de primero de Grado de Geografía y Gestión del Territorio de la Facultad de Geografía e Historia de la Universidad de Sevilla. Se trata de una asignatura de seis créditos contemplada dentro de la Formación Básica del Grado en el segundo cuatrimestre de primero. Se cuenta con la ventaja de la impartición de la asignatura en un aula informatizada en todos sus puestos. En relación al alumnado, el número de personas matriculadas era de 25, oscilando el número de asistentes a las clases durante todo el cuatrimestre entre los 15 y 20, un número que permitía, mediante la formación de pequeños grupos llevar a cabo esta experiencia docente.

Durante el curso pasado, dentro del primer ciclo de mejora de la fase de iniciación se diseñó una actividad con un apartado del citado tema (acercando al alumnado a través del uso de la fotografía en su entorno más cercano los contenidos de ese epígrafe, contaminación visual y fragilidad y calidad de un paisaje). Se planteó como ciclo de mejora parcial en este curso ampliar los contenidos a los de un tema completo aprovechando la experiencia del segundo ciclo de mejora del curso pasado, en el que se trató el paisaje como hilo conductor de una actividad de sintesis de toda la asignatura mediante un taller conceptual (Finkel, 2008).

\section{Diseño previo del Ciclo de Mejora Docente}

\section{Modelo metodológico.}

El modelo metodológico utilizado en los últimos cursos para el citado tema fue la impartición de clases magistrales y la realización de prácticas al final del tema. El modelo 
ideal sería uno basado en el principio de investigación en el que una serie de problemas vayan conduciendo a los alumnos hacía la construcción del conocimiento, cimentado sobre conocimientos previos. Debería mantenerse siempre el papel de guía del profesor, acompañando en ese aprendizaje al alumnado, protagonista del mismo (Bain, 2007). Por otro lado, los contenidos deben ser lo más cercanos posible a la realidad del alumnado y despertando, en lo posible, su interés y emociones. Deben valorarse los conocimientos iniciales y los posteriores a la resolución de los problemas desencadenantes de las sesiones para observar los cambios en su conocimiento. Las respuestas a los problemas deberían ser lo más complejas y completas posible, apoyando siempre la diversidad de opiniones y el debate, reflejándose, y esto es lo más complicado, todo este proceso en la fase de evaluación.

Por las características de la asignatura (de iniciación a contenidos de asignaturas de los cursos siguientes) se seleccionó para llevar a cabo la técnica del taller conceptual un tema, el dedicado al paisaje, que se encuentra descontextualizado del resto del temario. Además se trata de contenidos muy cercanos a problemas relevantes de la realidad local: la banalización del paisaje urbano por el uso turístico en un recorrido que realiza a diario del alumnado para acceder a la Facultad, su paisaje andado y vivido. Tiene, a su vez, implicaciones sociales y ambientales que van de lo local a lo global, por la manera en que se modifican los flujos turísticos en un mundo globalizado, la respuesta de las sociedades que los acogen y su reflejo en la conservación y transformaciones de su patrimonio paisajístico.

Una pregunta pondría en marcha el taller a modo de reto: ¿es compatible el uso turístico con la conservación del paisaje histórico de Sevilla? Para responderla, se les propone realizar un informe como expertos del comité 
nacional español de ICOMOS- Consejo de Monumentos, Conjuntos y Sitios del Patrimonio Histórico y Cultural- para el que precisaban manejar los contenidos del tema, intentando poner en relación las ideas y conocimientos ya adquiridos por el alumnado y sus emociones, al tratarse el lugar de estudio de un espacio cercano, con un reto temático con continuidad en distintas sesiones.

\section{Mapa de contenidos.}

Para llevar a cabo el ciclo de mejora se realizó una revisión de los contenidos que tradicionalmente se habían abordado en el tema dedicado al paisaje, generado un nuevo mapa de contenidos menos centrado en ciertos aspectos conceptuales y más dedicados a los procedimentales del citado tema. Esto permite el desarrollo de destrezas y habilidades y, en especial, la conexión con otros conocimientos aprendidos en el resto de la asignatura y la cursada en el primer cuatrimestre, Geografía Humana.

Jornadas de Formación e Innovación Docente del Profesorado | № 1 (2018) Esta obra se distribuye con la licencia Creative Commons 


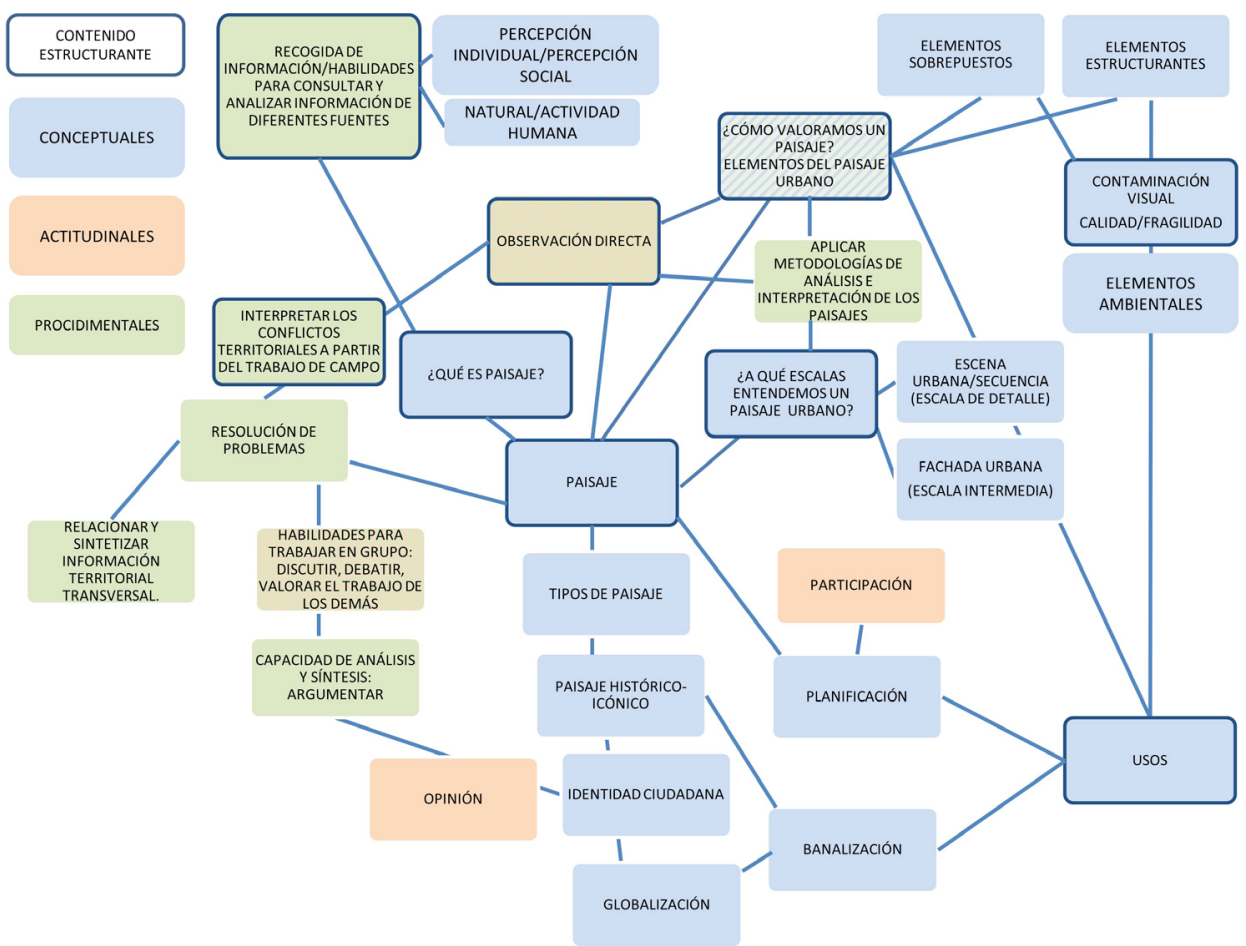

Figura1. Mapa de contenidos.

\section{Secuencia de actividades}

La secuencia de actividades y preguntas planteadas a partir de un primer problema de investigación (como se ha comentado previamente, relacionado con el estado actual y la sostenibilidad frente al uso turístico de los paisajes más cercanos y reconocibles por al alumnado, el eje Avenida de la Constitución-Plaza Nueva y la fachada del río a su paso por el casco histórico) los debía conducir a través de los contenidos del tema para poder responder finalmente al problema planteado en la pregunta inicial (¿es compatible el uso turístico con la conservación del paisaje histórico de Sevilla?). Se reservaba la mayoría de horas del ciclo para una salida de campo en la que contenidos conceptuales, procedimentales y actitudinales pudieron ponerse en comunicación de manera mucho más 
efectiva que en el aula, con recogida de información por parte del alumnado (apoyada por sesiones previas y posteriores en el aula) usando una cámara fotográfica y un cuaderno de campo, de la manera más autónoma posible y con la ayuda de glosarios.

Se intentó de esta manera organizar en las tres sesiones de trabajo actividades que estuvieran relacionadas con los siguientes contenidos de la asignatura:

Búsqueda de información, ordenando las distintas fases en el desarrollo del trabajo de campo y comenzando con la recopilación previa de información y datos (sesión 1).

2)

Recogida de información (observación directa, sesión

Tratamiento y análisis de la información recabada (sesión 3).

Cada una de las sesiones se apoyaba en la resolución de preguntas parciales (¿qué es el paisaje?, ¿cómo se valora?) dentro del reto que supone la resolución del problema inicial.

Jornadas de Formación e Innovación Docente del Profesorado I № 1 (2018) Esta obra se distribuye con la licencia Creative Commons 
Tabla 1 Cronograma de actividades desarrollado en el CM

\begin{tabular}{|c|c|c|}
\hline Sesión & Actividad & Descripción \\
\hline \multirow[t]{3}{*}{1} & 1 & $\begin{array}{l}\text { Entrega del cuestionario previo en el que se va- } \\
\text { lora través de diferentes niveles de aprendizaje para } \\
\text { los contenidos seleccionados el nivel de partida del } \\
\text { alumnado. }\end{array}$ \\
\hline & 2 & $\begin{array}{l}\text { Presentación del taller conceptual en clase, forma- } \\
\text { ción de grupos y planteamiento del problema, asig- } \\
\text { nación de roles dentro de cada grupo. }\end{array}$ \\
\hline & 3 & $\begin{array}{l}\text { Presentación de la pregunta guía de la sesión. De- } \\
\text { finición de paisaje: uso de internet, buscadores es- } \\
\text { pecializados y guías relacionadas con la búsqueda } \\
\text { bibliográfica en un aula informatizada, alternándose } \\
\text { momentos de trabajo individual con la práctica de la } \\
\text { escucha activa dentro del grupo. }\end{array}$ \\
\hline \multirow[t]{4}{*}{2} & 1 & $\begin{array}{l}\text { Contextualización de la fase recogida de información } \\
\text { usando nubes de palabras generadas por cada grupo } \\
\text { al comienzo de la salida de campo. } \\
\text { Presentación de la pregunta que guía la secuencia de } \\
\text { actividades de la sesión: cómo valorar un paisaje e } \\
\text { identificar los elementos que lo componen. }\end{array}$ \\
\hline & 2 & Análisis de una fachada urbana. \\
\hline & 3 & Análisis de una secuencia. \\
\hline & 4 & Análisis de una escena urbana. \\
\hline \multirow[t]{4}{*}{3} & 1 & $\begin{array}{l}\text { Puesta en común en el aula de los materiales ge- } \\
\text { nerados en las actividades previas mediante la pro- } \\
\text { ducción de un informe-matriz DAFO (Debilidades, } \\
\text { Amenazas, Fortalezas y Oportunidades) y un álbum } \\
\text { fotográfico (evaluable dentro del apartado de par- } \\
\text { ticipación de la evaluación continua) de la salida, } \\
\text { así como unas recomendaciones que debían reali- } \\
\text { zar como expertos del comité nacional español de } \\
\text { ICOMOS. }\end{array}$ \\
\hline & 2 & $\begin{array}{l}\text { Exposición por grupos de las conclusiones del in- } \\
\text { forme, siendo comentadas por el resto del alumnado. }\end{array}$ \\
\hline & 3 & $\begin{array}{l}\text { Debate final relacionado con la sostenibilidad y los } \\
\text { usos: resolución del problema. }\end{array}$ \\
\hline & 4 & $\begin{array}{l}\text { Entrega del cuestionario utilizado de manera previa } \\
\text { al taller para valorar los cambios en los conocimien- } \\
\text { tos del alumnado para los distintos niveles de apren- } \\
\text { dizaje sugeridos para cada contenido vertebrador. }\end{array}$ \\
\hline
\end{tabular}




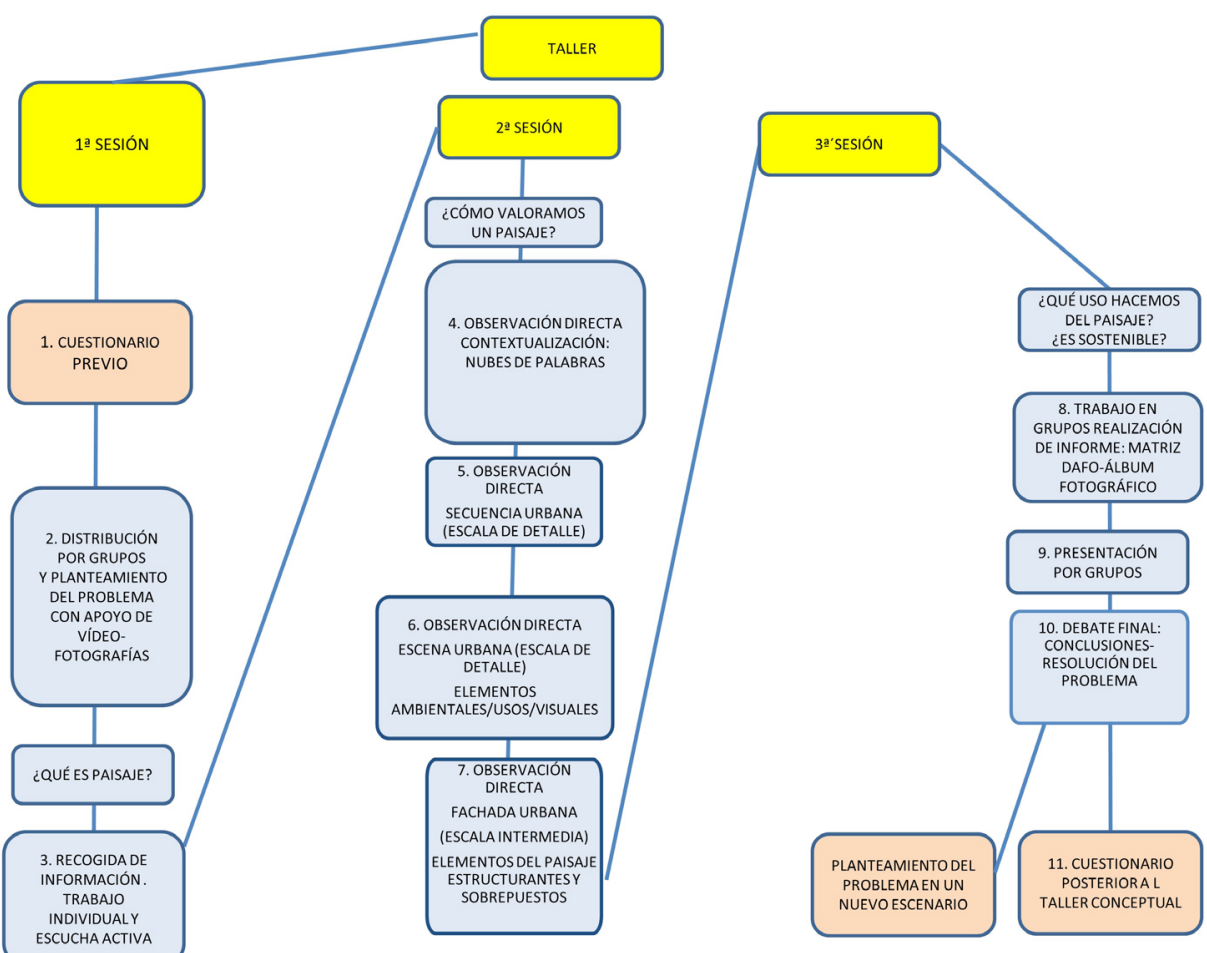

Figura 2. Secuencia de actividades.

\section{Aplicación del Ciclo de Mejora Docente}

\section{Relato resumido de las sesiones}

Como ya se ha comentado, se guio al alumnado a través de experiencias diversas (Finkel, 2008) con la herramienta denominada taller conceptual hasta una serie de conclusiones que acabasen interiorizando como propias. Las tuvieron que defender ante el resto de la clase, consiguiéndose una mayor implicación emocional a través del estudio de un entorno cercano, en interacción, con el mismo y con el grupo, interrelacionando los contenidos de la asignatura y siempre con el escenario de un problema de investigación.

Jornadas de Formación e Innovación Docente del Profesorado | № 1 (2018)

(c) Esta obra se distribuye con la licencia Creative Commons

Reconocimiento-NoComercial-SinObraDerivada

Internacional (CC BY-NC-ND 4.0.) 
Se intentó en esta fase utilizar las técnicas apropiadas para generar un aprendizaje crítico y la mayor relación posible con los contenidos de otros temas de la asignatura, dando validez a las distintas respuestas generadas al problema en los diferentes grupos.

Además se les planteó la posibilidad de realizar el trabajo de grupo de la asignatura siguiendo la metodología del taller. Lo podrían hacer en otro lugar sometido a las mismas presiones sobre el paisaje urbano en el casco histórico de Sevilla o en el entorno más cercano del alumnado (localidades o barrios en los que residan) justificando la necesidad del estudio por cambios de uso y presiones que no tienen que ser las relacionadas con el turismo.

Las sesiones programadas se desarrollaron como estaba previsto siendo los mayores inconvenientes la ausencia de algunos miembros del grupo en las sesiones previas y posteriores a la salida de campo que impedían un seguimiento completo de la actividad.

En la línea de las experiencias anteriores el alumnado manifestó su satisfacción por el uso del trabajo cooperativo, la estructura en pequeños hitos que ir superando, así como la realización del informe final de carácter profesional.

También destacó la consulta de diferentes fuentes y la correcta realización en un estilo seleccionado y reconocible de una cita y referencia bibliográfica como la actividad en que más surgió la autocorrección entre grupos.

Como cambio más importante se destaca la adaptación que se tuvo que realizar en la temporalización de la tercera sesión, que pasaron a ser dos, para aumentar el tiempo destinado por el grupo para la elaboración de conclusiones, así como para la puesta en común y el debate. 


\section{Evaluación del aprendizaje del alumnado}

El sistema de evaluación del aprendizaje utilizado fue el de las escaleras de aprendizaje, para conocer el punto de partida de los alumnos. Volviendo a los contenidos que aparecen en la figura 1, se seleccionaron los siguientes para la elaboración de los cuestionarios que debieron rellenar antes y después del taller:

Conceptos de Paisaje y calidad paisajística.

Realizar búsquedas bibliográficas con criterio académico.

Identificación de elementos del paisaje urbano.

Relacionar el paisaje con fenómenos como la turistización o la museificación y los cambios en la funcionalidad del entorno urbano.

Al objeto de elaborar escaleras de aprendizaje fundamentadas se han tomado las respuestas del alumnado que completó el cuestionario tanto en la fase inicial del taller como en la final. De este modo, trabajamos con un total de 26 cuestionarios al ser 13 los alumnos que asistieron a todas las sesiones.

A continuación se enumeran las preguntas del cuestionario, los posibles niveles de conocimiento según sus respuestas en la escalera de aprendizaje y el porcentaje de cuestionarios en cada una de los escalones antes y después de las sesiones.

Jornadas de Formación e Innovación Docente del Profesorado | № 1 (2018) Esta obra se distribuye con la licencia Creative Commons 
Tabla 2. Pregunta 1 del cuestionario y niveles de aprendizaje: Define qué es para ti el paisaje.

\begin{tabular}{|l|l|l|}
\hline $\begin{array}{l}\text { Nivel de } \\
\text { respuesta }\end{array}$ & Descripción & $\begin{array}{l}\text { Porcentaje de } \\
\text { respuestas }\end{array}$ \\
\hline Nivel 1 & $\begin{array}{l}\text { Definición imprecisa en la que no se en- } \\
\text { cuentran los componentes más genéricos } \\
\text { del paisaje. }\end{array}$ & $\begin{array}{l}\text { Pre: } 46 \% \\
\text { Post: 31\% }\end{array}$ \\
\hline Nivel 2 & $\begin{array}{l}\text { Aparece en la definición la importancia de } \\
\text { la componente natural, pero no la humana } \\
\text { o sus interrelaciones. }\end{array}$ & $\begin{array}{l}\text { Pre: 38\% } \\
\text { Post: 54\% }\end{array}$ \\
\hline Nivel 3 & $\begin{array}{l}\text { Define correctamente el concepto pero sin } \\
\text { terminología adecuada o entrar en aspectos } \\
\text { como la percepción individual. }\end{array}$ & $\begin{array}{l}\text { Pre: 15\% } \\
\text { Post: 8\% }\end{array}$ \\
\hline Nivel 4 & $\begin{array}{l}\text { Es consciente de que el paisaje incluye, } \\
\text { además de elementos tangibles, elemen- } \\
\text { tos intangibles, aspectos perceptuales del } \\
\text { individuo. }\end{array}$ & $\begin{array}{l}\text { Pre: 0\% } \\
\text { Post: 8\% }\end{array}$ \\
\hline
\end{tabular}

Tabla 3. Pregunta 2 del cuestionario y niveles de aprendizaje: Necesitas definir este concepto para un trabajo de la asignatura y acudes a varias fuentes, ¿cómo reflejarías esas fuentes en el trabajo?

\begin{tabular}{|l|l|l|}
\hline $\begin{array}{l}\text { Nivel de } \\
\text { respuesta }\end{array}$ & Descripción & $\begin{array}{l}\text { Porcentaje de } \\
\text { respuestas }\end{array}$ \\
\hline Nivel 1 & $\begin{array}{l}\text { Mediante el nombre de la web o la URL del } \\
\text { buscador, la búsqueda o la web. }\end{array}$ & $\begin{array}{l}\text { Pre: 31\% } \\
\text { Post: 8\% }\end{array}$ \\
\hline Nivel 2 & $\begin{array}{l}\text { Sabe que debe hacerlo mediante una cita o } \\
\text { referencia bibliográfica, pero no conoce nin- } \\
\text { gún estilo para hacerlo ni lo que necesita } \\
\text { para realizarlas. }\end{array}$ & $\begin{array}{l}\text { Pre: } 62 \% \\
\text { Post: 46\% }\end{array}$ \\
\hline Nivel 3 & $\begin{array}{l}\text { Identifica estilos pero no es capaz de hacer } \\
\text { la referencia. }\end{array}$ & $\begin{array}{l}\text { Pre: 8\% } \\
\text { Post: 31\% }\end{array}$ \\
\hline Nivel 4 & $\begin{array}{l}\text { Diferencia entre cita y referencia bibliográ- } \\
\text { fica, conoce al menos un estilo con el que } \\
\text { elaborarlas y los elementos necesarios para } \\
\text { construirlas. }\end{array}$ & $\begin{array}{l}\text { Pre: 0\% } \\
\text { Post: 15\% }\end{array}$ \\
\hline
\end{tabular}

Jornadas de Formación e Innovación Docente del Profesorado I № 1 (2018) Esta obra se distribuye con la licencia Creative Commons Reconocimiento-NoComercial-SinObraDerivada Internacional (CC BY-NC-ND 4.0.) 
Tabla 4. Pregunta 3 del cuestionario y niveles de aprendizaje: ¿Cuándo podemos destacar en un paisaje su calidad visual? Nombra varios ejemplos y explica brevemente por qué los has escogido.

\begin{tabular}{|l|l|l|}
\hline $\begin{array}{l}\text { Nivel de } \\
\text { respuesta }\end{array}$ & Descripción & $\begin{array}{l}\text { Porcentaje de } \\
\text { respuestas }\end{array}$ \\
\hline Nivel 1 & Utiliza ejemplos de paisajes naturales icónicos. & $\begin{array}{l}\text { Pre: } 85 \% \\
\text { Post:31\% }\end{array}$ \\
\hline Nivel 2 & $\begin{array}{l}\text { Utiliza ejemplos variados y cercanos pero sin justificar } \\
\text { el porqué. }\end{array}$ & $\begin{array}{l}\text { Pre: } 8 \% \\
\text { Post: } 15 \%\end{array}$ \\
\hline Nivel 3 & $\begin{array}{l}\text { Utiliza ejemplos variados y cercanos y justifica el grado } \\
\text { de excelencia de ese paisaje o mérito para no ser alte- } \\
\text { rado o destruido. }\end{array}$ & $\begin{array}{l}\text { Prest: } 31 \% \\
\text { Posto }\end{array}$ \\
\hline Nivel 4 & $\begin{array}{l}\text { Utiliza ejemplos justificados, variados y cercanos, re- } \\
\text { lacionándolos con otros conceptos como la fragilidad } \\
\text { paisajística o la contaminación visual. }\end{array}$ & $\begin{array}{l}\text { Pre: } 0 \% \\
\text { Post: } 23 \%\end{array}$ \\
\hline
\end{tabular}

Tabla 5. Pregunta 4 del cuestionario y niveles de aprendizaje: 3. ¿Si tuvieras que estudiar el paisaje de una plaza o calle, ¿en qué elementos o aspectos te fijarías?

\begin{tabular}{|c|c|c|}
\hline $\begin{array}{l}\text { Nivel de } \\
\text { respuesta }\end{array}$ & Descripción & $\begin{array}{l}\text { Porcentaje de } \\
\text { respuestas }\end{array}$ \\
\hline Nivel 1 & $\begin{array}{l}\text { Identifica elementos con vocabulario impreciso o } \\
\text { coloquial. }\end{array}$ & $\begin{array}{l}\text { Pre: } 62 \% \\
\text { Post: } 31 \%\end{array}$ \\
\hline Nivel 2 & $\begin{array}{l}\text { Identifica elementos del paisaje urbano como los usos, } \\
\text { las edificaciones, el plano o elementos sobrepuestos, } \\
\text { pero sin estructura o diferenciar en importancia. }\end{array}$ & $\begin{array}{l}\text { Pre: } 38 \% \\
\text { Post: } 54 \%\end{array}$ \\
\hline Nivel 3 & $\begin{array}{l}\text { Identifica elementos del paisaje urbano como los usos, } \\
\text { las edificaciones, el plano o elementos sobrepuestos, } \\
\text { en un discurso ordenado aunque aún no técnico. }\end{array}$ & $\begin{array}{l}\text { Pre: } 0 \% \\
\text { Post: } 15 \%\end{array}$ \\
\hline Nivel 4 & $\begin{array}{l}\text { Identifica elementos del paisaje urbano, los clasifica } \\
\text { dándoles importancia y subraya ciertos elementos vi- } \\
\text { suales ambientales o de utilización con un lenguaje } \\
\text { técnico. }\end{array}$ & $\begin{array}{l}\text { Pre: } 0 \% \\
\text { Post: } 0 \%\end{array}$ \\
\hline
\end{tabular}

Jornadas de Formación e Innovación Docente del Profesorado | № 1 (2018) Esta obra se distribuye con la licencia Creative Commons Reconocimiento-NoComercial-SinObraDerivada Internacional (CC BY-NC-ND 4.0.) 
Tabla 6. Pregunta 5 del cuestionario y niveles de aprendizaje: ¿Qué relación puede haber entre actividades como el turismo y el paisaje de tu ciudad?

\begin{tabular}{|l|l|l|}
\hline $\begin{array}{l}\text { Nivel de } \\
\text { respuesta }\end{array}$ & Descripción & $\begin{array}{l}\text { Porcentaje de } \\
\text { respuestas }\end{array}$ \\
\hline Nivel 1 & $\begin{array}{l}\text { Cree que hay relación pero sin razonar la } \\
\text { respuesta }\end{array}$ & $\begin{array}{l}\text { Pre: } 85 \% \\
\text { Post: 38\% }\end{array}$ \\
\hline Nivel 2 & $\begin{array}{l}\text { Identifica elementos que actúan en el pro- } \\
\text { ceso como las empresas multinacionales y } \\
\text { Su relación con la identidad ciudadana y la } \\
\text { alteración de paisajes icónicos. }\end{array}$ & $\begin{array}{l}\text { Pre: } 8 \% \\
\text { Post: } 46 \%\end{array}$ \\
\hline Nivel 3 & $\begin{array}{l}\text { Analiza la relación del proceso con los cam- } \\
\text { bios de uso y su afección en otros tipos de } \\
\text { patrimonio, no solo el arquitectónico }\end{array}$ & $\begin{array}{l}\text { Pre: } 8 \% \\
\text { Post: } 0 \%\end{array}$ \\
\hline Nivel 4 & $\begin{array}{l}\text { Entiende el proceso de banalización del pai- } \\
\text { saje y lo desarrolla con lenguaje técnico. }\end{array}$ & $\begin{array}{l}\text { Pre: } 0 \% \\
\text { Post: } 15 \%\end{array}$ \\
\hline
\end{tabular}

Teniendo en cuenta estos resultados, es la pregunta relacionada con el concepto de calidad paisajística donde ha habido una mejoría más notable, seguida por las preguntas relacionadas con los procedimientos de búsqueda de información y la valoración de la relación entre la actividad turística y el paisaje urbano. Las más teóricas (preguntas 1 y 4), relacionadas con el concepto de paisaje y los elementos que lo componen son en las que se observa una menor evolución. Por tanto, los avances han sido mayores en preguntas más relacionadas con destrezas (relacionadas con la búsqueda bibliográfica) y actitudes y valoración del el paisaje que las relacionadas con contenidos más conceptuales.

Para una evaluación global del aprendizaje del alumnado se podría agrupar las respuestas de las distintas preguntas a 4 posibles niveles de respuesta siguiendo este esquema:

Jornadas de Formación e Innovación Docente del Profesorado I № 1 (2018) Esta obra se distribuye con la licencia Creative Commons 
Tabla 7. Niveles de respuesta evaluación global del aprendizaje.

\begin{tabular}{|l|l|}
\hline $\begin{array}{l}\text { Nivel de } \\
\text { respuesta }\end{array}$ & Descripción \\
\hline Nivel 1 & Respuestas incorrectas o no razonadas. \\
\hline Nivel 2 & Respuestas incompletas y no estructuradas. \\
\hline Nivel 3 & $\begin{array}{l}\text { Respuestas correctas pero no completas para lo que } \\
\text { se espera tras el taller. }\end{array}$ \\
\hline Nivel 4 & $\begin{array}{l}\text { Respuestas más completas y cercanas a los conteni- } \\
\text { dos trabajados en el aula y en la salida de campo. }\end{array}$ \\
\hline
\end{tabular}

Con esta agrupación de respuestas podemos observar (figuras 3 y 4) que los porcentajes de contestaciones de los escalones inferiores, que se corresponden con los contenidos más básicos, se han reducido aunque en ningún caso ha llegado a hacerse nulo el porcentaje en el primer escalón. Sí se ha producido un notable trasvase de respuestas desde el escalón inferior a los intermedios, y tras el ciclo de mejora aparecen también respuestas en el escalón superior, inexistentes al principio de la tarea, el más complejo desde el punto de vista de los contenidos y las relaciones entre los mismos, por lo que podemos considerar positivos los resultados.

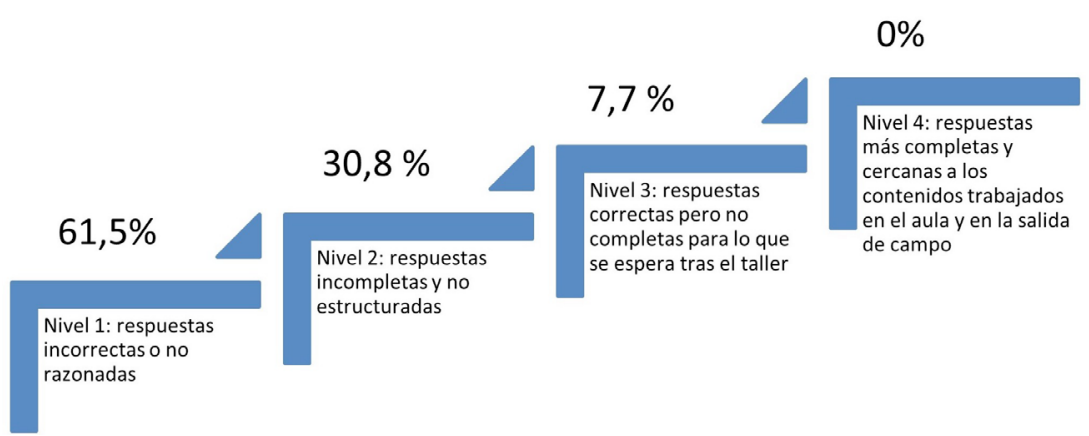

Figura 3: Porcen taje de respuestas de las distintas preguntas clasificadas por los 4 posibles niveles de respuesta en los cuestionarios previos.

Jornadas de Formación e Innovación Docente del Profesorado | № 1 (2018) Esta obra se distribuye con la licencia Creative Commons Reconocimiento-NoComercial-SinObraDerivada Internacional (CC BY-NC-ND 4.0.) 


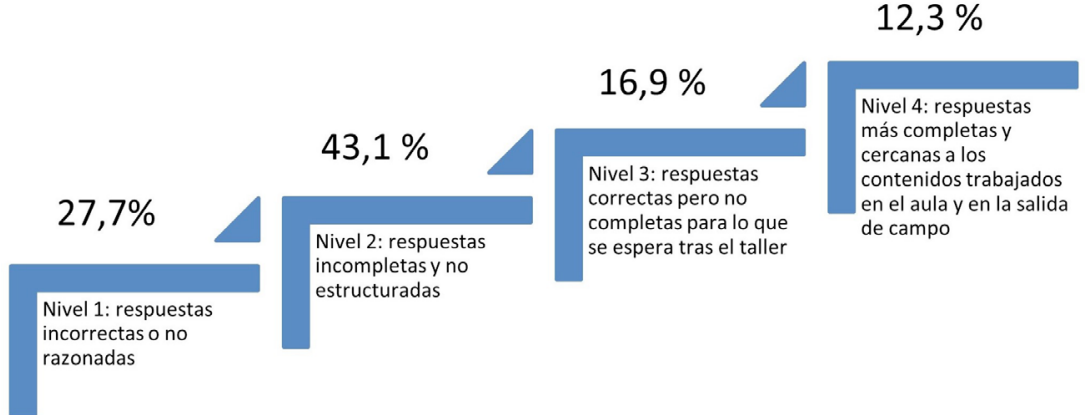

Figura 4: Porcentaje de respuestas de las distintas preguntas clasificadas por los 4 posibles niveles de respuesta en los cuestionarios finales.

\section{Evaluación del CMD.}

Por todo esto, se recomienda mantener la incorporación de la herramienta del taller conceptual para la práctica docente de ciertas unidades de la programación de la asignatura, dedicadas al desarrollo del trabajo de campo (recopilación previa de información y datos; recogida de información, encuestas, entrevistas, y búsquedas bibliográficas) y el análisis del paisaje a través de problemas que motiven al alumnado y de la creación de un producto final a través del trabajo cooperativo.

Para ello, debe tenerse en cuenta en la secuenciación una mayor temporalización de las sesiones dedicadas al debate final y la búsqueda de conclusiones y estudio de las soluciones y materiales generados por cada grupo, ya que es en este momento cuando se produce una mayor valoración del trabajo en equipo y el proceso de aprendizaje al acercarse más las sesiones a su propia experiencia y futura situación profesional. Para poder valorar más correctamente la metodología del taller sería interesante utilizar un sistema de rúbrica sobre el aprendizaje de contenidos conceptuales, procedimentales y actitudinales y los diferentes aspectos metodológicos del taller y del diario del profesor. 
Por otro lado, como aspectos importantes a incorporar a toda la experiencia docente habitual destacan el uso del mapa de contenidos para clarificar contenidos, tipos, y relaciones entre ellos y seleccionar los principales que deben centrar el taller, así como el uso de los cuestionarios de conocimientos evaluables mediante escaleras de aprendizaje para modificar en las sesiones la importancia y enfoque de los contenidos en los que centrar cada actividad.

Para concluir, considero que los principios didácticos que tras esta experiencia deben permanecer en mi práctica docente futura son el trabajo autónomo del alumnado y la necesidad de relacionar ese trabajo con su futuro profesional, convirtiéndose el aula en un espacio de reflexión, al tratarse de talleres con soluciones abiertas pero contextualizadas, fomentando el aprendizaje funcional y conectado con la realidad, la vida cotidiana y los intereses del alumnado, para la motivación e implicación del mismo en su propio aprendizaje.

\section{Referencias bibliográficas}

Bain, K. (2007). Lo que hacen los mejores profesores universitarios. Valencia: Publicaciones de la Universidad de Valencia.

Finkel, D. (2008). Dar clase con la boca cerrada. Valencia: Publicaciones de la Universidad de Valencia.

Porlán Ariza, R. (coord.) (2017). Enseñanza Universitaria. Cómo mejorarla. Madrid: Ediciones Morata. 